\title{
Mechanics and correlations of flow phenomena in intersecting ducts
}

\author{
S. Umeda, Wen-Jei Yang, T. Tanaka
}

Abstract Three kinds of experiments are conducted to determine flow characteristics inside two intersecting square ducts: flow visualization by means of the dye injection, hydrogen bubble and string methods, velocity measurement using the laser Doppler anemometry, and pressure measurement using a piezometer. Angle of intersection and Reynolds number are varied. Four distinct flow regimes are disclosed: entrance, initial centrifugal, intersecting and final centrifugal regions. The geometrical dividing line in the intersection zone of the two ducts forms a flow divider separating the flow network into two independent, symmetrical flow units. Each flow unit constitutes a curved passage with a "radius of curvature" whose magnitude varies with the angle of intersection. Hence, the secondary effects prevail in each unit, resulting in complete flow mixing. The mechanisms disclosed by the flow visualization and LDA methods are supported by the quantitative results from the piezometer measurements. The string method discloses an important evidence that the intersecting zone promotes turbulence. A location of the maximum energy grade and minimum hydraulic grade is disclosed on the flow divider where the surrounding streamlines congregate and disperse.

1

\section{Introduction}

Flow intersecting networks are abundant in nature and in some industrial systems. However, little effort has been directed towards the investigation of flow behavior in these intersecting networks.

Zhang et al. (1993a) measured pressure drops across various sections of a pneumatic flow network with one intersection. A semi-empirical model was proposed to correlate the pressure drop across the intersection zone with a flow divider separating the network into two independent, symmetrical flow units. Flow was assumed to undergo a sudden contraction into the zone followed by a sudden expansion out of it. Results compare very well with experiments. A flow visualization study (Zhang et al., 1992) of an intersecting duct system on a water table used the hydrogen bubble method. The study confirmed the existence of a flow divider in the intersection zone. This series of

Received: 12 October 1992/Accepted: 10 December 1993

S. Umeda

Department of Civil Engineering, Fukuyama University, Fukuyama, Japan

Wen-Jei Yang, T. Tanaka

Department of Mechanical Engineering and Applied Mechanics,

University of Michigan, Ann Arbor, MI 48109, USA investigations is currently extending into thermal behavior in the flow network (Zhang et al, 1993b). The merit of a flow network with multiple sequential intersection is a periodic disruption of flow which prevents the formation of a fully-developed boundary layer.

This paper employs a hydraulic model. Three programs are used to explore flow characteristics: flow visualization, LDV, and the pressure measurement. Both qualitative and quantitative information are obtained.

2

\section{Theoretical equations}

Flow inside the intersecting ducts is considered steady and incompressible, and all losses are accounted for (including duct friction, curvature, and changes in the flow cross-sectional area). Let the velocity within both the upper and lower tanks be zero. The velocity and pressure measurements are conducted at stations $2,3, \ldots, n-1(=8)$ using the LDV and the piezometer, respectively. The velocity and pressure at station $i$ are represented by $V_{i}$ and $P_{i}$, respectively. The application of Bernoulli's theorem yields the hydraulic head for the upper tank $H_{1}$ as

$$
\begin{aligned}
H_{1}= & p_{1} / \gamma+\left(V_{1}^{2}+\zeta_{1} V_{1}^{2}\right) /(2 g) \\
& \cdots \cdots \cdots \cdots \\
= & p_{i} / \gamma+\left(V_{i}^{2}+\zeta_{1} V_{1}^{2}+\cdots+\zeta_{i} V_{1}^{2}\right) /(2 g) \\
& \cdots \cdots \cdots \cdots \cdots \\
= & H_{2}+\left(\zeta_{1} V_{1}^{2}+\cdots+\zeta_{n} V_{n}^{2}\right) /(2 g)
\end{aligned}
$$

where $\gamma$ denotes the specific weight of the fluid and $\zeta_{i}$ is the loss coefficient of local dynamic head. Let us define, for $i=1,2, \ldots, n$,

$\alpha_{i}=\left(V_{i} / V\right)^{2}$

$\zeta_{i}=\alpha_{i} \zeta_{i}$

Here, $\mathrm{V}$ is the average of the left and right inlet velocities ( $V_{L}$ and $\left.V_{R}\right) \cdot \alpha_{i}$ signifies the ratio of the local to average dynamic heads. While $\zeta_{i}$ stands for the loss coefficient based on the local dynamic head, $\zeta_{i}$ is that based on the average dynamic head of the entire flow passage. Both $K_{z}$ and $K_{q}$ are a measure of loss of local dynamic head during the flow.

Now, Eq. (1) can be rewritten as

$$
\begin{aligned}
H_{1}= & p_{1} / \gamma+\left(\alpha_{1}+\zeta_{1}\right) V^{2} /(2 g) \\
& \cdots \cdots \cdots+\cdots \\
= & p_{i} / \gamma+\left(\alpha_{1}+\xi_{1}+\cdots+\xi_{i}\right) V^{2} /(2 g) \\
& \cdots \cdots \cdots \cdots \\
= & H_{2}+\left(\xi_{1}+\cdots+\xi_{n}\right) V^{2} /(2 g)
\end{aligned}
$$


The pressure hard loss at station $i, \Delta p_{i}$, is defined as $\left(p_{i-1}-p_{i}\right) / \gamma$. Then, one obtains

$$
\begin{aligned}
\Delta P_{1}= & H_{1}-p_{1} / \gamma=\left(\alpha_{1}+\xi_{1}\right) V^{2} /(2 g) \\
& \ldots \ldots \cdots \cdots \cdots \\
\Delta P_{i}= & \left(p_{i-1}-p_{i}\right) / \gamma=\left(\alpha_{i}-\alpha_{i-1}+\xi_{i}\right) V^{2} /(2 g) \\
& \cdots \cdots \cdots \cdots \cdots \cdots \\
\Delta P_{n}= & \left(p_{n-1}-p_{n}\right) / \gamma-H_{2}=\left(-\alpha_{n-1}+\xi_{n}\right) V^{2} /(2 g)
\end{aligned}
$$

The above equations are combined to yield the total pressure head loss as

$$
\Delta P_{t}=\sum_{i=1}^{n} \Delta P_{i}=H_{1}-H_{2}=\sum_{i-1}^{n} \xi_{i} V^{2} /(2 g)
$$

Let $h$ denote the dynamic head defined as

$h=V^{2} /(2 g), \quad h_{i}=V_{i}^{2} /(2 g)$

The above equations can be rearranged to yield

$$
\begin{aligned}
\zeta_{1}= & \Delta P_{1} / h_{1}-1.0 \\
& \ldots \ldots \ldots \\
\zeta_{i}= & \left(\Delta P_{i}+h_{i-1}\right) / h_{i}-1.0 \\
& \ldots \ldots \ldots \ldots \ldots \\
\zeta_{n}= & \left(\Delta P_{n}+h_{n-1}\right) / h_{n}
\end{aligned}
$$

and

$$
\begin{aligned}
\zeta_{1}= & \left(\Delta P_{1}+h_{1}\right) / h \\
& \ldots \ldots \ldots \\
\zeta_{i}= & \left(\Delta P_{i}-h_{i}+h_{i-1}\right) / h \\
& \ldots \ldots \ldots \ldots) \\
\zeta_{n}= & \left(\Delta P_{n}+h_{n-1}\right) / h
\end{aligned}
$$

The total loss coefficient over the entire flow passage, $K_{z}$ can be obtained from Eq. (7) as

$K_{z}=\sum_{i=1}^{n} \zeta_{i}=\sum_{i=1}^{n} \Delta P_{i} / h_{i}+K_{v}-n+1$

where

$K_{v}=\sum_{i=1}^{n-1}\left(V_{i} / V_{i+1}\right)^{2}$

Similarly, the sum of all $\xi_{i}^{\prime} \mathrm{s}, K_{q}$, can be found from Eq. (8) as

$K_{q}=\sum_{i=1}^{n} \xi_{i}=\sum_{i=1}^{n} \Delta P_{i} / h_{i}$

The sum of the products of the pressure head loss ratio, $\Delta P_{i} / \Delta P_{t}$, and that the dynamic head ratio is defined as a new parameter $K_{c}$ as

$K_{c}=\sum_{i=1}^{n}\left[\Delta P_{i}\left(V / V_{i}\right)^{2} / \Delta P_{t}\right]$

It can be rewritten in terms of $K_{z}, K_{q}$ and $K_{v}$ as

$$
\begin{aligned}
K_{c} & =\sum_{i=1}^{n}\left[\left(\Delta P_{i} / \alpha_{i}\right) / \Delta P_{t}\right] \\
& =\left(K_{z} / K_{q}\right)-\left(K_{v}-n+1\right) / K_{q}
\end{aligned}
$$

Being related to the head loss $\Delta P_{t}$, pressure drop $\Delta P_{i}$ and velocity coefficient $\alpha_{i}, K_{c}$ represents the characteristics of both pressure and velocity changes and thus can be considered a friction coefficient. An attempt will be made later in Sect. 4.3 to
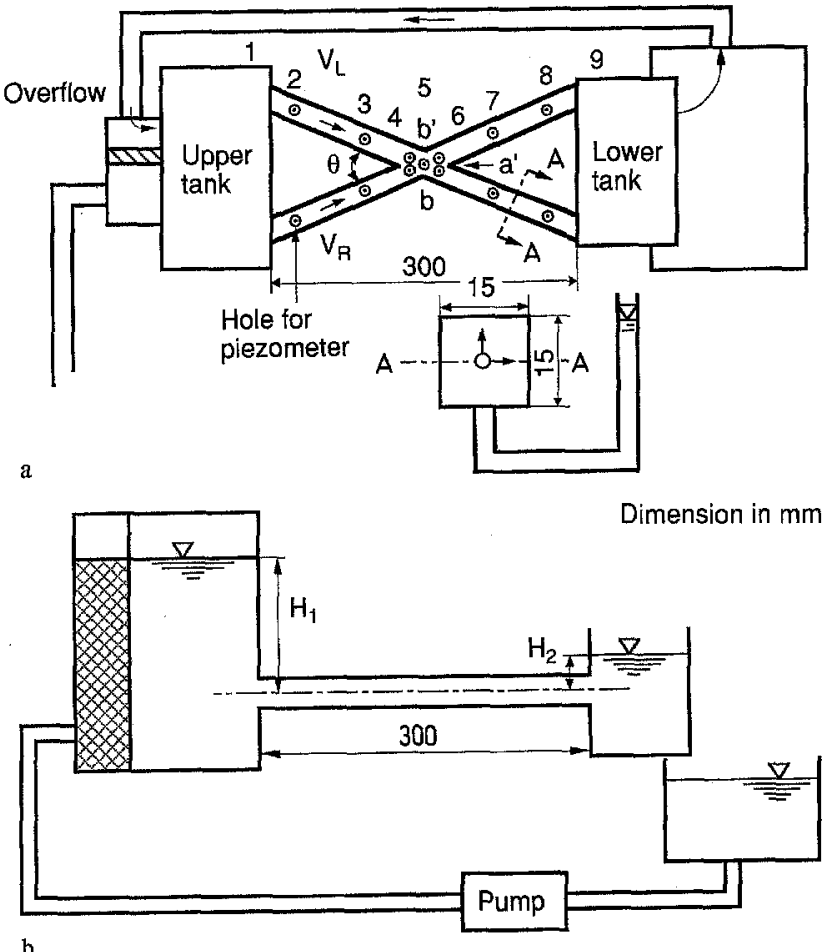

Fig. 1a, b. A schematic of experimental setup. a Top view; b side view

use $K_{c}$ in correlating flow characteristics inside the intersecting flow passages.

\section{3}

\section{Experimental apparatus}

Figure 1 depicts a schematic of a hydraulic recirculating flow system for testing flow inside two intersecting square ducts, (a) top view and (b) side view. The flow network was placed between two head tanks with a distance of $30 \mathrm{~cm}$. The water level in the upper tank was maintained at a higher head of $H_{1}$, and the lower tank at a lower head of $H_{2}$, The difference $\left(H_{1}-H_{2}\right)$ caused a flow through the two ducts with an intersection angle of $\theta$. The flow was recirculated by means of a pump. Each duct was square, $1.5 \mathrm{~cm} \times 1.5 \mathrm{~cm}$, in cross section and made of acrylic for illumination and observation. Three intersection angles were tested: $30^{\circ}, 60^{\circ}$ and $90^{\circ}$. Both the flow rate and pressure in the network were varied by changing the magnitude of $\left(\mathrm{H}_{1}-\mathrm{H}_{2}\right)$. Small holes were drilled at strategic locations on the bottom of the flow passages for pressure measurements using a piezometer. Numbers 1 through 9 in Fig. 1 indicate the location of such measurements. The flow velocity was measured at various locations by means of a laser Doppler anemometry. Both the intersecting angle and flow velocity were varied.

\section{4}

\section{Experimental results}

Three kinds of experiments were conducted: flow visualization, LDV and pressure measurement. Test cases for symmetrical flow are listed in Table 1.

\section{1}

\section{Flow visualization}

Three flow visualization techniques were employed: dye injection, hydrogen bubble, and string methods. 
Table 1. Test cases for symmetrical flow

\begin{tabular}{|c|c|c|c|c|c|}
\hline Run & $\theta$ & $H_{1}, \mathrm{~cm}$ & $H_{2}, \mathrm{~cm}$ & $V, \mathrm{~cm} / \mathrm{s}$ & $R e$ \\
\hline 1 & $30^{\circ}$ & 2.7 & 2.1 & 22.5 & 3380 \\
\hline 2 & & 4.6 & 3.2 & 32.8 & 4920 \\
\hline 3 & & 11.9 & 2.2 & 96.7 & 14510 \\
\hline 4 & & 14.1 & 2.1 & 107.8 & 16160 \\
\hline 5 & $60^{\circ}$ & 4.1 & 2.2 & 35.6 & 5330 \\
\hline 6 & & 8.3 & 2.3 & 63.6 & 9540 \\
\hline 7 & & 14.4 & 2.2 & 97.7 & 14650 \\
\hline 8 & & 17.3 & 2.0 & 110.4 & 16560 \\
\hline 9 & $90^{\circ}$ & 5.4 & 2.0 & 48.7 & 7300 \\
\hline 10 & & 8.4 & 2.0 & 71.5 & 10720 \\
\hline 11 & & 14.3 & 2.0 & 95.4 & 14300 \\
\hline 12 & & 18.2 & 2.0 & 106.1 & 15910 \\
\hline
\end{tabular}

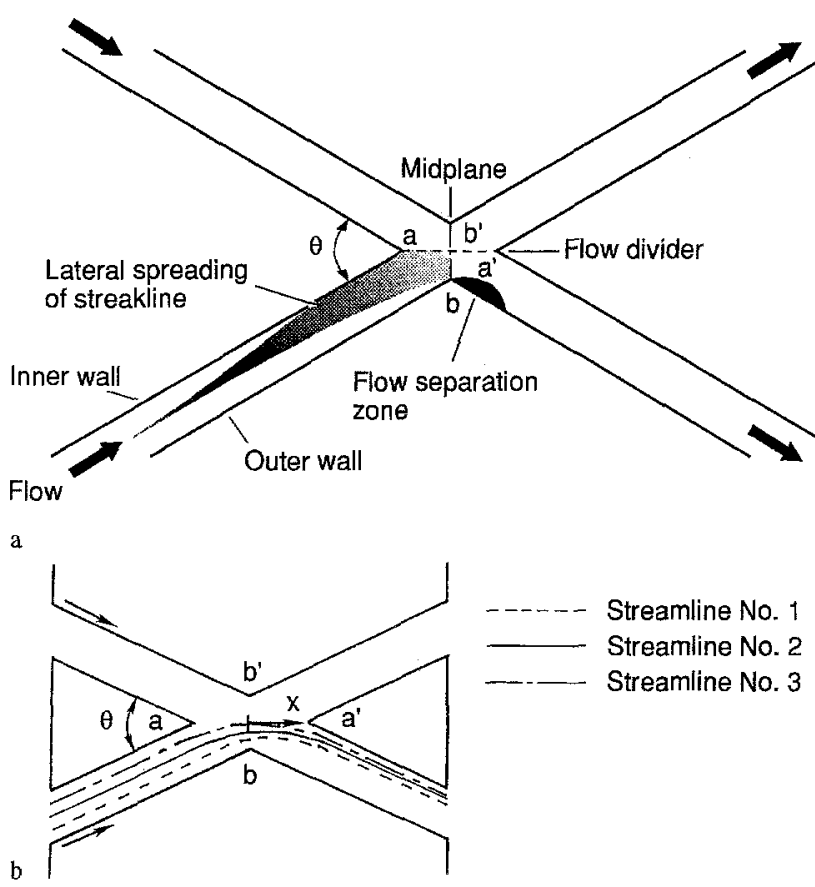

Fig. 2a, b. A schematic of flow behavior revealed by dye injection method. a Flow behavior; $b$ streamlines

\subsection{1}

\section{Dye injection method}

Two dyes were injected in lines into two entrance ports of a network: blue water and Rhodamine $B$ which produce blue and red colors, respectively. Figure 2 (a) is a schematic of flow behavior revealed by the dye injection method. The flow is from left to right as indicated by the arrows. It is observed that the longitudinal plane of symmetry connecting the two intersection points of the inner duct walls, $a-a^{\prime}$, separates the flow in the left-side duct (facing the flow direction) from that in the right-side duct. Note that there are two planes of symmetry in the intersection zone. The other lateral plane of symmetry (to be called "midway") connects the two intersection points of the outer duct walls, $b-b^{\prime}$. This divider appears in all cases irrespective of the intersection angle and flow rate. However, at lower flow rates, one flow (mostly in the left duct) sometimes crosses the divider into the other (right duct), whenever there is a slight unbalance in the flow rates between the two ducts (mostly higher in the left duct). The second disclosure is that at a certain distance from the entrance, each dye streak line begins to spread out and shift toward the inner duct wall. Figure $2 b$ is a schematic of streamlines disclosed by the dye injection method. Streamline No. 2 (solid line) passes through the center of the upstream duct, while streamlines No. 1 (broken line) and 3 (chain line) pass the midway between the duct center and the outer and inner duct walls, respectively. It is observed that all streamlines, after passing the midway $b-b^{\prime}$, are pushed outward toward the inner wall in the downstream duct by the centrifugal force. This results in the formation of a flow separation region (i.e., vortex) on the outer duct wall beginning at the intersection point $b$.

The vortex shape differs depending upon the intersection angle, the upper tank head (i.e., flow velocity), and the extent of asymmetrical flow in the two ducts. The change in the vortex shape is small in the $\theta=30^{\circ}$ case. This indicates that there is a force in the duct pushing the flow toward the inner wall side. The third and most important observation is the formation of a flow separation region on each outer duct wall beginning at the "midway" plane, $b-b$ ', as shown in Fig. 2.

\subsection{2}

\section{Hydrogen bubble method}

The hydrogen bubbles were generated in line series at the entrance ports of both ducts. These bubbles form a smooth, curved steakline around the pointed corner in the intersection zone. As the flow rate or intersection angle is increased, the centrifugal force on the bubbles will be enhanced and the bubble streakline will move closer to the outer wall. The formation of a flow separation zone was observed (not shown) with the accumulation of bubbles in the region. Sometimes these bubbles aggregated to form a larger one there. It is of interest to note that the bubble or bubbles in the flow separation region were the last to disappear after the bubble generator was turned off.

\subsection{3}

\section{String method}

A string in each duct was fixed at the entrance port. Two CCD cameras were positioned at downstream from the intersecting region, one for top view and the other for side view, to record the motion of either one string. Since the top and side views of string motion differ in response to a change in the upper tank head, $H_{1}$, the time and coordinate location were recorded when the string was at its highest and lowest positions in each view. The fast Fourier transformation (FFT) was then operated on the string motion to determine its power spectrum and auto-correlation coefficient.

Like a difference in the vortex shape due to an asymmetrical flow in the two ducts, the oscillation of the two strings in the ducts also differs. In both the top and side views, one observes very intense string oscillations in the downstream duct passages. One typical result for the top view of an asymmetrical flow is depicted in Fig. 3 for $\theta=60^{\circ}, H_{l}=11.0 \mathrm{~cm}, H_{r}=40.5 \mathrm{~cm}$, and $\mathrm{H}_{2}=7.9 \mathrm{~cm}$. Note that this experiment was conducted in an intersecting flow network of $3.0 \mathrm{~cm}$ square ducts, allowing the strings to have ample space for oscillations. It is disclosed that when the streamlines take a bulged shape or a vortex is 


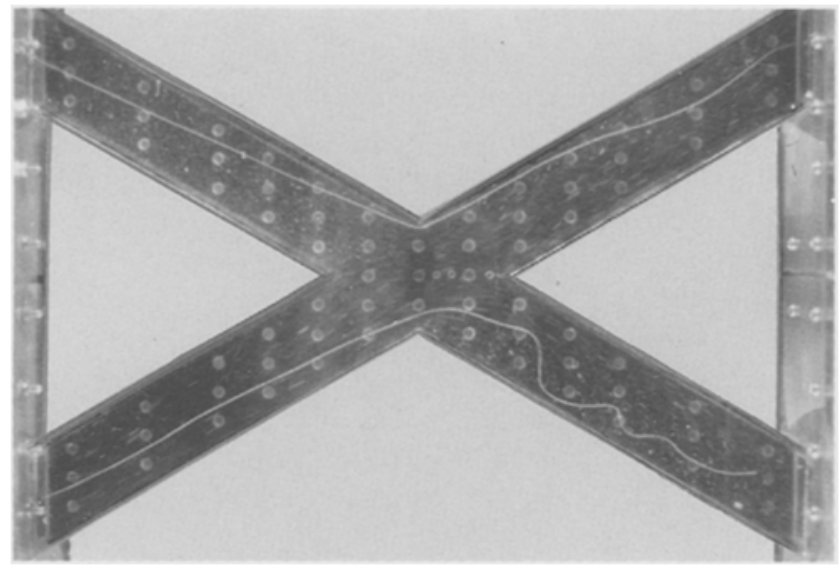

Fig. 3. Visualization of flow oscillation by means of the string method, for $\theta=60^{\circ}$ and top view, with $H_{l}=11.0 \mathrm{~cm}, H_{r}=40.5 \mathrm{~cm}$ and $H_{2}=7.9 \mathrm{~cm}$
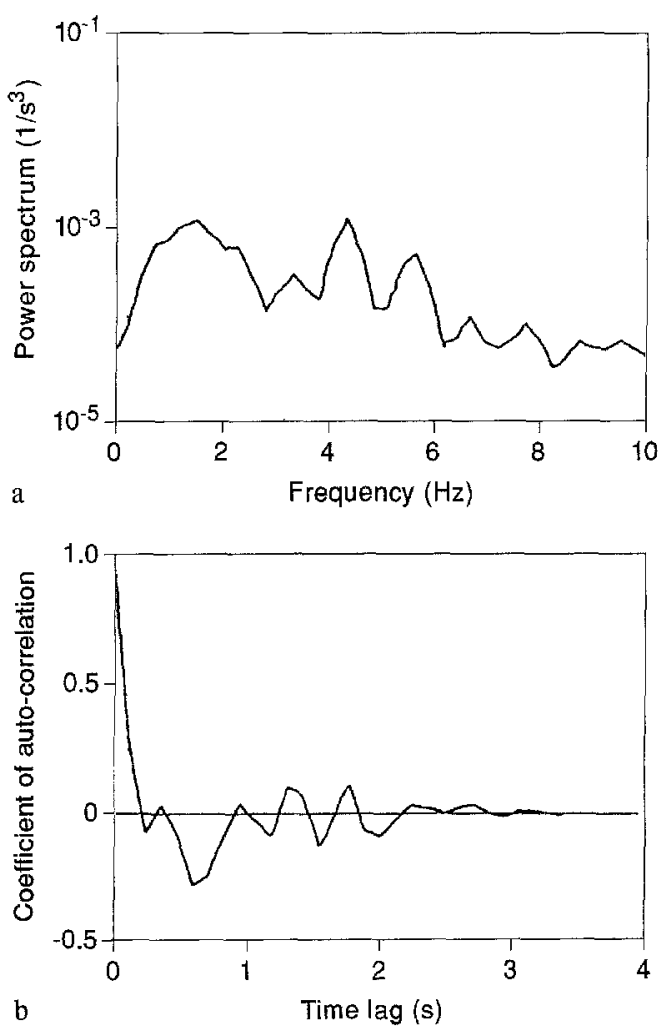

Fig. 4a, b. Results of fast Fourier transformation on string oscillation in Fig. 3. for $\theta=60^{\circ}$ and top view, with $H_{I}=11.0 \mathrm{~cm}, H_{r}=40.5 \mathrm{~cm}$ and $\mathrm{H}_{2}=7.9 \mathrm{~cm}$. a Power spectrum and $\mathbf{b}$ coefficient of auto-correlation

formed in a downstream passage due to the centrifugal effect, the string tends to vigorously oscillate with a larger amplitude.

Figure 4 presents FFT results of Fig. 3. It is observed that a relatively high power specturm prevails over a substantial frequency range, exhibiting the spectral characteristics of white noise. The coefficient of auto-correlation, shown in Fig. $4 \mathrm{~b}$ diminishes to zero rapidly. The test result for the side view shows a similar oscillation as that described above for the top view. In the case of a small intersecting angle or symmetrical flow, the power spectrum is high only at small frequencies and diminishes with an increase in the frequency. An important conclusion

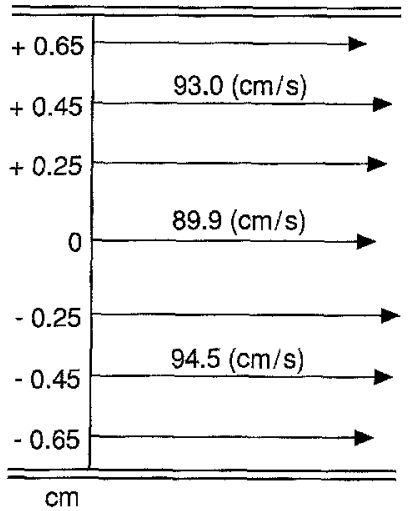

Fig. 5. Typical vertical velocity distribution at channel center measured by LDV method

is derived from this experiment that string oscillations exhibit the energy spectral characteristics of turbulent flow downstream from the intersection zone in the flow passage with the formation of a vortex. Thus, a very high heat transfer performance in the intersecting flow network (Zhang et al,, 1993b) can be attributed to this cause.

\section{2}

LDV

The origin of the coordinates $(x, y)$ is fixed at the center of the flow-cross section, with $x$ measuring the horizontal distance and $y$ the vertical length. LDV measurements were conducted through the top duct wall. Figure 5 shows a representative result of the vertical velocity profile of a duct, cross-sectioned at $x=0$. Since the velocity distribution is almost uniform, the center velocity at $y=0$ was taken as the mean value across the vertical cross-section on the $x=0$ midplane. LDV measurements were made at all locations with piezometer holes at $x=0$ to obtain the horizontal velocity distribution on the $y=0$ midplane. Only one portion of the horizontal velocity distribution is presented in Figs. $6 \mathrm{a}, \mathrm{b}$ and $\mathrm{c}$ for the intersecting angles of $30^{\circ}, 60^{\circ}$, and $90^{\circ}$, respectively. Each figure is supplemented by two sub-figures on each side of the intersecting ducts, showing velocity vector distributions in different parts of the intersecting region. The top sub-figure of Fig. 6a shows the velocity profiles at the midway $b-b^{\prime}$ (at station 5) and near the entrance to the left downstream duct. The bottom sub-figure includes two velocity profiles in the upstream half of the intersecting zone (the upper one for flow from the left duct and the lower one for flow from the right duct) and one velocity profile in the downstream half of the intersecting zone (at station 6 for flow into the right downstream duct). Those sub-figures in both Fig. $6 \mathrm{~b}$ and $c$ present the velocity profiles at three lateral cross sections at stations 4,5 and 6 in the intersecting zone shown in the main figure. The arrow direction indicates a flow into or out from a flow passage. Along the flow divider in the intersection zone, three sets of measurements were made on the vertical velocity distribution: at 3 locations $(-0.65,0,+0.65 \mathrm{~cm})$ from the flow divider; at 5 locations $(-0.65,-0.45,0,+0.45,+0.65 \mathrm{~cm})$; and at 7 locations $(-0.65,-0.45,-0.25,0,+0.25,+0.45$, $+0.65 \mathrm{~cm}$ ).

It is seen in the intersection zone that the velocity near the flow divider (which was disclosed by flow visualization) is low at 

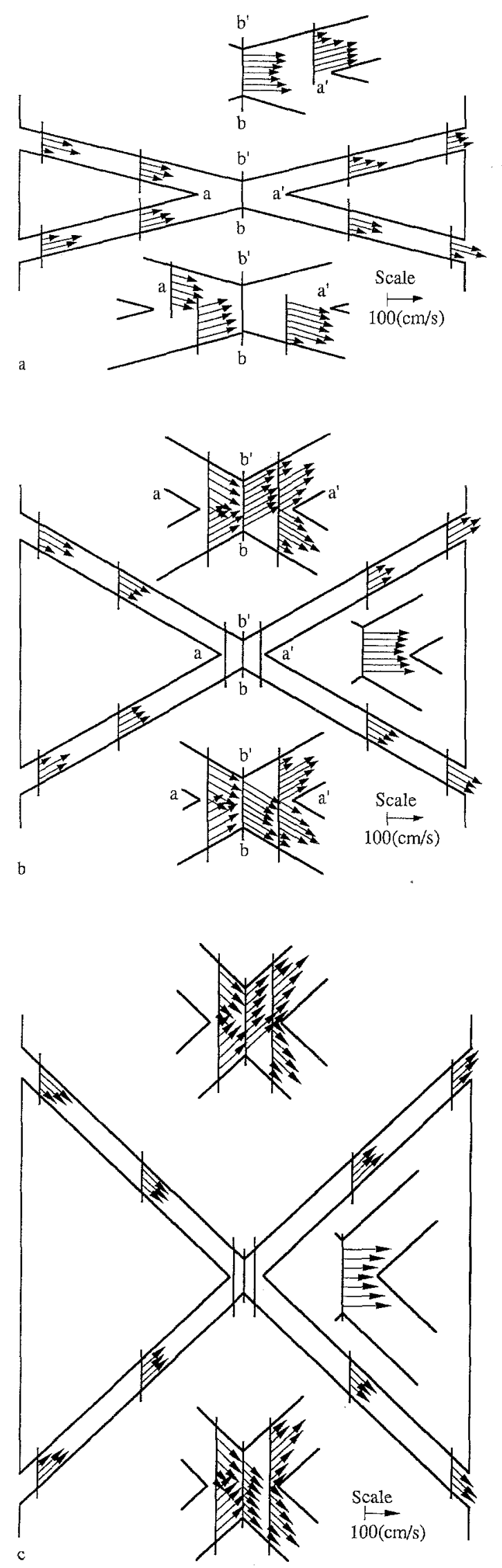

Fig. 6a-c. Horizontal velocity distribution at channel center measured by LDV method in the intersecting ducts of a Run No. $3, \theta=30^{\circ}$, b Run No. 7 , $\theta=60^{\circ}$, and $\mathrm{c}$ Run No. $11, \theta=90^{\circ}$ degrees the center and increases toward both the right and left ducts, where the flow separation regions are located. This observation confirms the disclosure of flow separation regions by flow visualization. Irrespective of the intersection angle and flow rate, flow velocity on the inner wall side is always lower than that on the outer wall side in the entrance region. This is because the outer wall corner has a larger angle than the inner wall corner, making it easier for the fluid to enter around the corner. The velocity difference diminishes downstream. This portion of the flow passage is called the entrance region. As the fluid approaches the intersection zone, the centrifugal force begins to take effect, because the stream is forced to turn in the presence of the flow divider, $a-a^{\prime}$. This is evidenced in the dispersion of the injected dye in the flow visualization experiment. This portion of the flow passage, from the end of the entrance region to the upstream edge $a$, is called the "initial centrifugal region".

The LDV measurements downstream of the inner wall intersection edge $a$, in Figs. $6 \mathrm{~b}$ and $\mathrm{c}$ respectively, indicate that these two streams have identical velocity profiles crossing each other at an angle of $\theta$. The horizontal velocity distribution on the "midway" plane, $b-b^{\prime}$, is symmetrical with respect to $a-a^{\prime}$, having a small dent at the flow divider. The flow upstream from the "midway" plane is practically uniform, while that downstream takes a substantial reduction in the velocity near the outer wall, evidence of a flow separation region. The collision of two streams having identical momentum causes the formation of a flow divider at $a-a^{\prime}$ plane, which in turn causes each stream to bend its flow direction and switch into the other duct. This curved flow induces a centrifugal force to produce the secondary flow effect. The centrifugal force effect prevails in both the right and left duct succeeding the intersection zone, resulting in a velocity profile with higher velocity near the inner duct wall, exactly opposite the velocity profile in the entrance region. This portion of the flow passage is referred to as the "final centrifugal region" which may extend beyond the ducts, as disclosed in a previous study (Zhang et al., 1992a). In summary, four distinct flow regimes are identified. No fully developed flow of any kind ever exists inside the flow network, irrespective of the intersection angle and flow rate.

\section{3}

\section{Pressure measurements}

Small holes for piezometers were drilled along the center line on the bottom surface of both ducts, as shown in Fig. 1. Additional holes were prepared in the intersection zone, one on each side of the flow divider at the midpoint between the divider and the duct walls. Local velocity $V_{i}$ was obtained by means of LDV, from which the average over the flow passage $V$ is calculated. With reference to the local velocity, pressure readings from the manometer yielded the pressure drop and head loss.

Only a few representative results are presented here to illustrate flow behavior. Figures $7 a, b$, and $c$ illustrate the variations of the energy grade, hydraulic grade, velocity coefficient, pressure drop, and head loss, along the left flow passage for the intersecting angles of 30,60 , and $90^{\circ}$, respectively. Note that energy grade is the sum of hydraulic grade and velocity head.

The location of all pressure measurements is marked on each figure. The domain of the four flow regimes can easily be identified by an examination of the pressure drop curve, because 

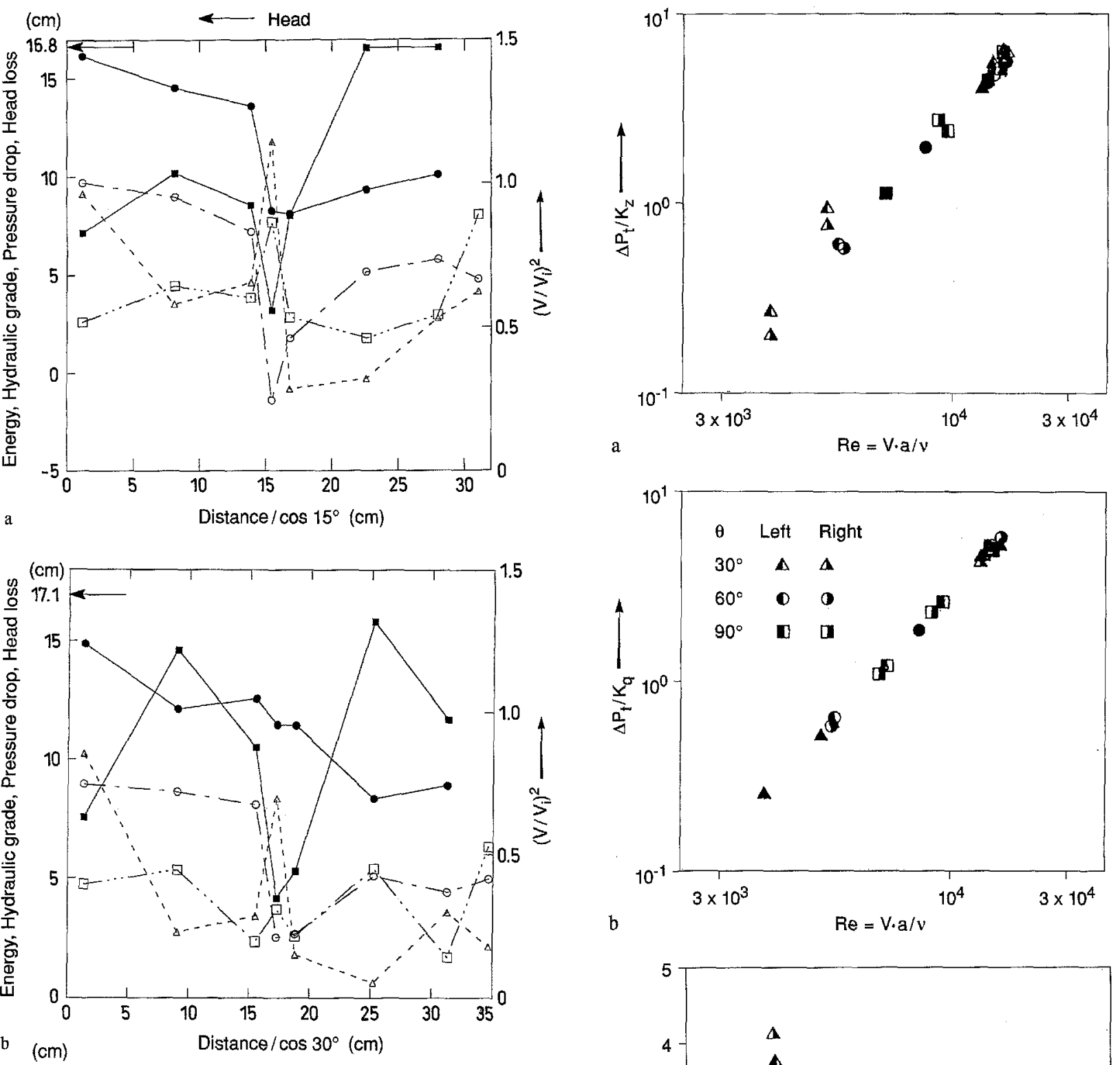

b

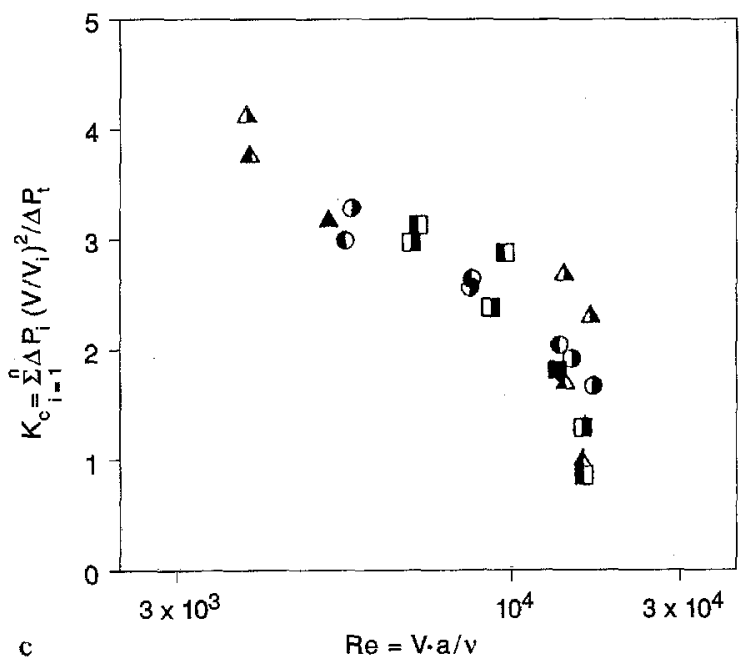

Fig. 8a-c. Effects of $R e$ on a $\Delta P_{t} / K_{z}$, b $\Delta P_{t} / K_{q}, \mathrm{c}$ and $K_{c}$

it is most sensitive and reflects different flow behavior in each regime. For example, in Fig. $7 \mathrm{~b}$, pressure drop continues in the entrance regime, as in all straight tubes and ducts. It begins to recover at the second piezometer hole, suggesting the end of the entrance region and the onset of the second regime. Then, a drastic change in the pressure drop characterizes the

Fig. $7 a-c$. Distribution of energy grade $\boldsymbol{\theta}$, hydraulic grade $O,\left(V / V_{i}\right)^{2} \mathbf{\square}$, pressure drop $\triangle$ and head loss $\square$ along the flow in the intersecting ducts of a $\theta=30^{\circ}$ b $\theta=60^{\circ}$, and c $\theta=90^{\circ}$ degrees 
intersection zone, with one maximum followed by one minimum. Thereafter comes the fourth flow regime in which the pressure head continues to recover, with a dent in a few rare occasions.

In all three intersection angles, the hydraulic grade line and pressure drop in the intersecting zone vary appreciably with a change in the overall head difference $\left(H_{1}-H_{2}\right)$. In Fig. $7 \mathrm{a}$ for $\theta=30^{\circ}$, the hydraulic grade at the midplane of the intersecting zone rises abruptly, with the pressure drop exceeding the energy grade line. The head loss varies significantly at the exit of the intersecting zone, while the velocity coefficient is minimally affected. The overall behavior of the head loss and the velocity coefficient is rather similar in the networks with $\theta=60$ and $90^{\circ}$.

The quantities which are theorectically derived in Eqs. (9) through (12) are plotted against the Reynolds number $R e$ in Fig. 8. $R e$ is defined as $V d / v$, where $d$ is the hydraulic diameter of the ducts and $v$ denotes the kinematic viscosity of water. Since the ratio $\Delta P_{t} / K_{q}$ expresses the mean flow velocity within the entire duct, its magnitude increases linearly with $R e$, as seen in Fig. 8b. The experiment reveals how the velocity variation induced by the total pressure head is related to $R e$. The $\Delta P_{t} / K_{z}-R e$ relationship for $\theta=60^{\circ}$ is a straight line on a $\log$-log plot (Fig. 8a). In addition to velocity change, the definition of $K_{z}$ involves the effect of variation in the energy grade line. This effect is small in the system of $\theta=60^{\circ}$, but is appreciable in the cases of $\theta=30$ and $90^{\circ}$, as evidenced by a deviation of the test data from the straight line. Figure $8 \mathrm{C}$ illustrates that $K_{c}$ decreases with an increase in $R e$ linearly when $\theta=60^{\circ}$. However, because of variations in both the velocity coefficient and the energy grade, $K_{c}$ for $\theta=30$ and $90^{\circ}$ changes more drastically with $R e$, implying more susceptability to the effect of flow change across the intersecting region. This result suggests that $K_{c}$ may measure the roughness within the intersecting ducts.

\section{5}

\section{Discussion}

It is disclosed from the study that flow in intersecting ducts undergoes a complete mixing in the intersection zone, as observed in the dye flow visualization. The main cause is a collision of two streams from the ducts, resulting in the formation of a flow divider in the intersection zone. The divider, in turn, forces each stream to curve and flow into the opposite duct. The centrifugal force resulting from a bent flow induces the secondary flow effect, such as the formation of flow separation zones on the duct walls downstream from the intersection of two outer walls. The centrifugal force (per unit mass) $F$ can be expressed as

$F=V_{\theta} 2 / R$

The tangential velocity $V_{\theta}$ is proportional to the flow rate and the radius of "curvature" $R$ depends on the angle of intersection, $\theta$. A larger $\theta$ means a smaller $R$ and vice versa. The strength of the collision of two symmetrical steams (with the same flow rate) is also dependent upon both the flow rate (which determines the collision velocity) and the intersection angle (which is the angle of collsion). When there is a flow disturbance (such as turbulence) or a slight unbalance in the two flow rates, the flow divider is broken, resulting in the crossing of one stream into the other.
An examination of Fig. 7 leads to the disclosure of a location on the flow divider $a-a^{\prime}$ at a short distance from the midway plane $b-b^{\prime}$ where energy grade is the maximum and hydraulic grade is the minimum. The location varies depending upon the intersection angle and corresponds to the place where streamlines near the flow divider on both sides congregate followed by dispersion, as confirmed by flow visualization. It is envisioned that the kinetic energy carried by conversing streams is assembled there and then dissipated downstream from there along the streamlines. The location can thus be considered "energy supply point" whose feature of the maximum flow velocity (as confirmed by an LDV measurement) and the minimum pressure is exactly opposite to that of a stagnation point (which is characterized by zero velocity and maximum pressure). It is a very interesting and important location in understanding the flow characteristics inside the intersecting zone, where the centrifugal and rumming effects coexist.

\section{6}

\section{Conclusions}

Flow visualization techniques have been employed to determine the steady flow characteristics of an incompressible fluid inside intersecting square ducts. The dye injection method has revealed the formation of a flow divider, flow separation regions, and a tendency for the dye to disperse toward the inner duct walls (at a higher flow rate or large intersection angle). The hydrogen bubble method has confirmed these findings. The LDV technique has been applied to measure local velocity distributions. Four flow regimes have been identified. The formation of the flow divider has been attributed to a collision of two symmetrical streams in the intersection zone. An examination of the variation of local velocity profiles has led to the conclusion that the presence of a flow divider forces flow streams to curve, producing a centrifugal force and the resulting secondary flow phenomena. The pressure measurements have provided both quantitative evidence of the existence of the four flow regimes and an explanation of flow behavior in each regime (most descriptive in the pressure-drop distribution curve). The fast Fourier transformation on string motion has revealed turbulent flow characteristics downstream from the intersecting zone. In addition, variations of the pressure drop, loss coefficients, and velocity coefficient with repsect to the Reynolds number are disclosed for the flow networks with different intersecting angles.

A new, hydrodynamically interesting, location is disclosed on the "flow divider" downstream from the "midway plane." It can be considered "energy supply point" where energy grade reaches the maximum value. The location characterized by the maximum energy grade and minimum hydraulic grade is precisely opposite to the feature of a stagnation point with zero velocity and maximum pressure.

\section{References}

Zhang N; Yang W-J; Xu Y; Lee CP (1992) An application of hydrogen bubble method to flow networks, Flow Visualization VI (eds: Y Tanida, H Miyashiro), Springer-Verlag, Berlin, 90-96

Zhang N; Yang W-J; Xu Y; Lee CP (1993a) Flow characteristics in flow networks. Exp Fluids 14: 25-32

Zhang N; Yang W-J; Xu Y; Lee CP (1993b) Heat transfer and friction loss performance in flow networks of multiple intersections. Experimental Heat Transfer (in print) 